\title{
$\omega$-JOINTLY METRIZABLE SPACES
}

\author{
M. A. AL SHUMRANI
}

\begin{abstract}
A topological space $X$ is $\omega$-jointly metrizable if for every countable collection of metrizable subspaces of $X$, there exists a metric on $X$ which metrizes every member of this collection. Although the Sorgenfrey line is not jointly partially metrizable [3], we prove that it is $\omega$-jointly metrizable.

We show that if $X$ is a regular first countable $T_{1}$-space such that $X$ is the union of two subspaces one of which is separable and metrizable, and the other is closed and discrete, then $X$ is $\omega$-jointly metrizable.
\end{abstract}

\section{INTRODUCTION}

Let $X$ be a topological space, and let $\mathcal{F}$ be a family of subspaces of $X$. We say that $X$ is jointly metrizable on $\mathcal{F}$, or $\mathcal{F}$-metrizable, if there is a metric $d$ on the set $X$ such that $d$ metrizes all subspaces of $X$ which belong to $\mathcal{F}$, that is, the restriction of $d$ to $A$ generates the subspace topology on $A$, for any $A \in \mathcal{F}$ (see $[1,2,3]$ ). In particular, $X$ is jointly partially metrizable, or a JPM-space, if there is a metric $d$ on $X$ which metrizes all metrizable subspaces of $X$.

Theorem 1.1. [3] Every regular first countable jointly partially metrizable $T_{1}$-space $X$ is metrizable.

Proposition 1.2. [3] If all metrizable subspaces of a space $X$ are discrete, then $X$ is jointly partially metrizable.

If $X$ is a space with no non-trivial convergent sequences, then all metrizable subspaces of $X$ are discrete, and hence, $X$ is a $J P M$-space. Therefore, every extremally disconnected Hausdorff space is a $J P M$-space. In particular, the Stone-Cech compactification $\beta(\omega)$ of the discrete space $\omega$ of natural numbers is jointly partially metrizable.

\section{2. $\omega$-JOINTLY METRIZABLE SPACES}

We say that a topological space $X$ is $\omega$-jointly metrizable if for every countable collection $\left\{M_{i}: i=1,2, \ldots\right\}$ of metrizable subspaces of $X$, there exists a metric $d$ on $X$ which metrizes every $M_{i}$ for $i=1,2, \ldots$.

Clearly, every $J P M$-space is $\omega$-jointly metrizable. 


\section{A. AL SHUMURANI}

Proposition 2.1. Suppose that $X$ is a space and any union of countably many metrizable subspaces of $X$ is metrizable. Then $X$ is $\omega$-jointly metrizable.

Proof. Let $\Gamma=\left\{M_{i}: i=1,2, \ldots\right\}$ be a countable family of metrizable subspaces of $X$. We show that there is a metric $d$ on $X$ which metrizes each $M_{i}$ for $i=1,2, \ldots$ Let $A=\cup M_{i}$. Then $A$ is metrizable. Let $d_{A}$ be the metric on $A$. We may assume that $d_{A}(x, y) \leq 1$ for $x, y \in A$. We define a metric $d$ on $X$ as follows: $d(x, y)=d_{A}(x, y)$ if $x, y \in A$; otherwise, we put $d(x, y)=1$ if $x$ and $y$ are distinct. It is clear that $d$ is a metric on $X$ which metrizes $A$ and thus it metrizes every subspace $M_{i}$ in $\Gamma$.

Observe that a subspace of the Sorgenfrey line is metrizable if and only if it is countable. Therefore, it follows from Proposition 2.1 that the Sorgenfrey line is $\omega$-jointly metrizable. On the other hand, the Sorgenfrey line is not jointly partially metrizable by Theorem 1.1, since it is first countable, Tychonoff, and not metrizable.

Example 2.2. Consider the space $A(n)$, the Alexandroff compactification of any uncountable discrete space $X$ [4, Example 3.5.14]. All subspaces of $A(n)$ are of the form $A(m)$ or $D(m)$ with $m \leq n$ and $A(m)$ is metrizable if $m$ is countable.

Let $\Gamma=\left\{M_{i}: i=1,2, \ldots\right\}$ be a countable family consisting of metrizable subspaces of $A(n)$. Let $B$ be the union of the metrizable subspaces $M_{i}$ of the form $A(m)$. Then $B$ is of the form $A(m)$ with $m$ countable and thus it is metrizable. Let $e$ be a metric on $B$. We may assume that $e(x, y) \leq 1$ for all $x, y \in B$. We define a metric $d$ on $A(n)$ as follows: $d(x, y)=e(x, y)$ if $x, y \in B$; otherwise, we put $d(x, y)=1$ if $x$ and $y$ are distinct. We can show that $d$ is a metric on $A(n)$ which metrizes $B$, any subspace $M_{i}$ of $B$ and metrizes any discrete subspace $D(m)$. Hence, $A(n)$ is $\omega$-jointly metrizable.

A natural question arises: under what conditions a space $X$ is $\omega$-jointly metrizable?

Now we present the main result of this article which gives conditions under which a space $X$ is $\omega$-jointly metrizable.

Theorem 2.3. Suppose that $(X, T)$ is a regular first countable $T_{1}$-space such that $X=A \cup B$. If $A$ is a closed discrete subspace and $B$ is a separable metrizable subspace, then $X$ is $\omega$-jointly metrizable.

Proof. Let $\Gamma$ be any countable collection $\left\{Y_{i}: i=1,2, \ldots\right\}$ of metrizable subspaces of $X$.

Let $Y$ be any member of $\Gamma$. Let $Y_{B}=Y \cap B$, and let $Y_{c}$ be the closure of $Y_{B}$ in the metrizable space $Y$ and $Y_{d}=Y \backslash Y_{c}$. 


\section{$\omega$-JOINTLY METRIZABLE SPACES}

Claim 1. $Y_{c}$ is a closed separable metrizable subspace of $Y$.

It is clear that $Y_{c}$ is closed and metrizable. It remains to show that it is separable. Since $B$ is separable metrizable, $B$ is second countable. It follows that $Y_{B}$ is second countable. Thus, $Y_{B}$ is separable, that is, there exists a countable subset $W$ of $Y_{B}$ such that $\bar{W} \supseteq Y_{B}$. Therefore, $\bar{W} \supseteq Y_{c}$. Hence, $Y_{c}$ is separable.

Next, the following statements are easily verified.

Claim 2. $Y_{d}$ is a closed discrete subspace of $Y$.

$\underline{\text { Claim 3. }} Y_{c} \cap Y_{d}=\emptyset$ and $Y_{c} \cup Y_{d}=Y$.

Claim 4. $Y_{c}$ and $Y_{d}$ are disjoint open subspaces of the space $Y$.

Now, let $A_{Y}=Y_{c} \cap A$.

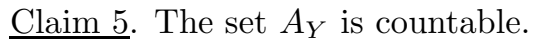

By Claim 1, the space $Y_{c}$ is second countable. Thus, $A_{Y}$ is second countable so that it is separable. But $A_{Y}$ is a discrete space being a subspace of $A$. Hence, $A_{Y}$ is countable.

Let $C$ be a countable subset of $A$ and let $S$ be a base for the topology $T$ of $X$. Let $S_{C}=S \cup\{\{x\}: x \in A \backslash C\}$. The family $S_{C}$ is a base of some topology $T_{C}$ on the set $X$.

Claim 6. The space $\left(X, T_{C}\right)$ is metrizable.

It is clear that $\left(X, T_{C}\right)$ is a $T_{1}$-space. We show that $\left(X, T_{C}\right)$ is regular. Let $x \in X$. Then $x \in B$ or $x \in C$ or $x \in A \backslash C$. If $x \in B$ or $x \in C$, then given any neighborhood $U$ of $x$, there exists a neighborhood $V$ of $x$ such that $\bar{V} \subset U$ since $(X, T)$ is regular. However, if $x \in A \backslash C$, then given any neighborhood $U$ of $x$, for the neighborhood $\{x\}$ we have $\{x\}=\overline{\{x\}} \subset U$. Hence, $\left(X, T_{C}\right)$ is regular. Let $V_{1}$ be a countable base for $B$. At every point $x \in C$, take a countable local base $V_{2}^{x}$. Let $V_{2}=\cup\left\{V_{2}^{x}: x \in C\right\}$. Then $V_{2}$ is countable. For $A \backslash C$, let $V_{3}$ be the family consisting of all singletons $\{x\}$ where $x \in A \backslash C$. Let $V=V_{1} \cup V_{2} \cup V_{3}$. Then $V$ is a $\sigma$-discrete base for the space $\left(X, T_{C}\right)$. Hence, the space $\left(X, T_{C}\right)$ is metrizable by the Bing Metrization Theorem [4, Theorem 4.4.8].

MISSOURI J. OF MATH. SCI., SPRING 2016 


\section{A. AL SHUMURANI}

Claim 7. Suppose that $A_{Y} \subset C$. Then the topology $T$ of $X$ induces on $Y$ the same topology as the topology $T_{C}$ does.

Let $T_{Y}$ denote the topology of $Y$ induced by $T$ and let $T_{C_{Y}}$ denote the topology of $Y$ induced by $T_{C}$. From the definition of $T_{C}$, it follows that $T_{Y} \subset T_{C_{Y}}$. Now we show that $T_{C_{Y}} \subset T_{Y}$. Take any $x \in A \backslash C$; then $\{x\}$ is a basis element in $T_{C_{Y}}$. Then $x \in Y_{d}$ and $x \notin Y_{c}$. There exists a basis element $U \in T$ containing $x$ such that $(U \cap Y) \cap Y_{B}=\emptyset$. That is, $(U \cap Y) \cap B=\emptyset$. Thus, $x \in U \cap Y \subset A$. Then $U \cap Y$ is an open discrete subspace of $\left(Y, T_{Y}\right)$. Hence, $\{x\} \in T_{Y}$. Therefore, $T_{C_{Y}} \subset T_{Y}$. Hence, the claim is proved.

Now let $d$ be a metric on $X$ that induces the topology $T_{C}$ of $X$. Let $C=\cup\left\{A_{Y_{i}}: i \in \omega\right\}$. Then $C$ is countable. Using Claim 7, we deduce that $d$ is the metric on $X$ which metrizes each metrizable subspace $Y_{i}$ of $\Gamma$. Hence, $X$ is $\omega$-jointly metrizable.

The following are examples of $\omega$-jointly metrizable spaces.

Example 2.4. Let $X$ be the Niemytzky plane [4, Example 1.2.4]. Then $X$ is a first countable Tychonoff space and $X=A \cup B$, where $A$ is the closed discrete bottom line and $B$ is the separable metrizable open half plane. Therefore, $X$ is $\omega$-jointly metrizable, by Theorem 2.3 .

Example 2.5. Let $X$ be the Mrowka space [4, Exercise 3.6.I]. Then $X$ is a first countable Tychonoff space and $X=A \cup B$, where $A$ is an uncountable closed discrete subspace of $X$ and $B$ is a countable open discrete subspace of $X$. Therefore, it follows from Theorem 2.3 that $X$ is $\omega$-jointly metrizable.

Example 2.6. Consider the set of real numbers $\mathbb{R}$ with the Rational Sequence Topology [5, Example 65]. With this topology, $\mathbb{R}=\mathbb{P} \cup \mathbb{Q}$, where $\mathbb{P}$ is the set of irrationals and $\mathbb{Q}$ is the set of rationals, is regular $T_{1}$-space and first countable. $\mathbb{P}$ is a closed discrete subspace and $\mathbb{Q}$ is an open separable metrizable subspace. Therefore, it follows from Theorem 2.3 that $\mathbb{R}$ with the Rational Sequence Topology is $\omega$-jointly metrizable.

We shall now give an example of a space which is not $\omega$-jointly metrizable.

Example 2.7. Consider the Michael line [4, Example 5.1.32]. Let $Y=$ $\mathbb{P} \cup\{q\}$ be a subset of the real line where $\mathbb{P}$ is the set of irrationals and $q$ is a rational number. Then $Y$ is a metrizable subspace of the real line and thus $\{q\}$ is a $G_{\delta}$-set in $Y$. Using Exercise 5.5.2 in [4], we conclude that $Y$ is a metrizable subspace of the Michael line.

Claim. If $x$ is a real number and $A$ is a subset of the Michael line such that $x \in \bar{A}$, then either $x \in \overline{A \cap \mathbb{P}}$ or $x \in \overline{A \cap \mathbb{Q}}$, where $\mathbb{Q}$ is the set of rationals. 


\section{$\omega$-JOINTLY METRIZABLE SPACES}

Note that $A=A \cap \mathbb{R}=A \cap(\mathbb{P} \cup \mathbb{Q})$. Then $A=(A \cap \mathbb{P}) \cup(A \cap \mathbb{Q})$. Thus,


$x \in \overline{A \cap \mathbb{P}}$ or $x \in \overline{A \cap \mathbb{Q}}$.

Now we show that the Michael line is not an $\omega$-jointly metrizable. Assume the contrary. Consider the countable family of metrizable subspaces $\Gamma=\{\mathbb{P} \cup\{q\}: q \in \mathbb{Q}\} \cup\{\mathbb{Q}\}$. Then there exists a metric $d$ on the Michael line metrizing each member of $\Gamma$. Next, we show that $d$ metrizes the Michael line. Let $x$ be any real number and $A$ any subset of the Michael line. We shall show that $x \in \bar{A}$ if and only if $d(x, A)=0$.

First, assume that $x \in \bar{A}$. By the above Claim, either $x \in \overline{A \cap \mathbb{P}}$ or $x \in \overline{A \cap \mathbb{Q}}$. Suppose that $x \in \overline{A \cap \mathbb{P}}$. Let $C=(A \cap \mathbb{P}) \cup\{x\}$. If either $x$ is rational or irrational, then $C$ is metrized by $d$ since $C$ is subset of $\mathbb{P} \cup\{x\}$. Thus, $d(x, A \cap \mathbb{P})=0$. Hence, $d(x, A)=0$. Suppose that $x \in \overline{A \cap \mathbb{Q}}$. Let $C=(A \cap \mathbb{Q}) \cup\{x\}$. Since $\overline{A \cap \mathbb{Q}} \subset \overline{\mathbb{Q}}$ and $\mathbb{Q}$ is closed, it follows that $x \in \mathbb{Q}$. Thus, $C$ is metrized by $d$ since $C$ is subset of $\mathbb{Q}$. Therefore, $d(x, A \cap \mathbb{Q})=0$. Hence, $d(x, A)=0$.

Conversely, assume that $d(x, A)=0$. We shall show that $x \in \bar{A}$. Assume that $x \notin \bar{A}$. Then $x \notin A$. For each positive $n \in \omega$, fix $a_{n} \in A$ such that $d\left(x, a_{n}\right)<1 / n$. Let $B=\left\{a_{n}: n \in \omega\right\}$. Then $B$ is an infinite subset of $A$ and $d(x, B)=0$. Note that $B=(B \cap \mathbb{P}) \cup(B \cap \mathbb{Q})$ is infinite. Then either $B \cap \mathbb{P}$ or $B \cap \mathbb{Q}$ is an infinite subset of $B$. Assume that $B \cap \mathbb{P}$ is an infinite subset of $B$. Then $d(x, B \cap \mathbb{P})=0$. Let $C=(B \cap \mathbb{P}) \cup\{x\}$. If either $x$ is rational or irrational, $C$ is metrized by $d$ since $C$ is subset of $\mathbb{P} \cup\{x\}$. Since $B \cap \mathbb{P} \subset B \subset A$ and $x \notin \bar{A}$, it follows that $x \notin \overline{B \cap \mathbb{P}}$. Therefore, $d(x, B \cap \mathbb{P})>0$ which is a contradiction. Hence, $x \in \bar{A}$. Assume that $B \cap \mathbb{Q}$ is an infinite subset of $B$. Then $d(x, B \cap \mathbb{Q})=0$. Let $C=(B \cap \mathbb{Q}) \cup\{x\}$. If $x$ is rational, then $C$ is metrized by $d$ since $C \subset \mathbb{Q}$. Since $B \cap \mathbb{Q} \subset B \subset A$ and $x \notin \bar{A}$, it follows that $x \notin \overline{B \cap \mathbb{Q}}$. Therefore, $d(x, B \cap \mathbb{Q})>0$ which is a contradiction. Hence, $x \in \bar{A}$. However, if $x$ is irrational, then $x$ is isolated in the Michael line. Thus, $x$ is isolated in every subspace of the Michael line containing $x$. Therefore, $d(x, B \cap \mathbb{Q})=\inf \{d(x, y): y \in B \cap \mathbb{Q}\}>0$ which is a contradiction. Hence, $x \in \bar{A}$.

\section{REFERENCES}

[1] A. V. Arhangel'skii and M. A. Al Shumrani, Jointly partially metrizable spaces, C. R. Acad. Bulg. Sci., 65.6 (2012), 727-732.

[2] A. V. Arhangel'skii and M. A. Al Shumrani, Joint metrizability of spaces on families of subspaces, and a metrization theorem for compacta, Proceedings of International Conference on Topology and its Applications (ICTA2011, Islamabad, Pakistan, July 4-10, 2011), Cambridge Scientific Publishers, 2012, pp. 1-9.

[3] A. V. Arhangel'skii, M. M. Choban, and M. A. Al Shumrani, Joint metrizability of subspaces and perfect mappings, Topology and its Applications, 169 (2014), 2-15.

[4] R. Engelking, General Topology, PWN, Warszawa, 1977.

MISSOURI J. OF MATH. SCI., SPRING 2016 


\section{A. AL SHUMURANI}

[5] L. A. Steen and J. A. Seebach, Jr., Counterexamples in Topology, Dover Publications, Inc. New York, 1995.

MSC2010: 54A25, 54B05

Key words and phrases: $J P M$-space, $\omega$-jointly metrizable space

Department of Mathematics, King Abdulaziz University, P. O. Box 80203, Jeddah 21589, Saudi Arabia

E-mail address: maalshmrani1@kau.edu.sa 